\title{
VPS33B mutations cause ARKID syndrome affecting Rab protein interaction, collagen modification and epidermal structure
}

\author{
Gruber $\mathbf{R}^{1}$, Rogerson $C^{2}$, Windpassinger $C^{3}$, Strohal $R^{4}$, Schmuth $M^{1}$, Kroisel $P^{3}$, Janecke $A R^{5}$, Gissen $P^{2}$ \\ ${ }^{1}$ Department of Dermatology, Venereology and Allergology, Medical University of Innsbruck, Austria; ${ }^{2}$ MRC Laboratory for Molecular Cell \\ Biology, University College London, UK; ${ }^{3}$ Institute of Human Genetics, Medical University of Graz, Austria; ${ }^{4}$ Department of Dermatology, \\ Academic Teaching Hospital Feldkirch, Austria; ${ }^{5}$ Department of Pediatrics I, Medical University of Innsbruck, Austria
}

Aut.-Rec. Keratoderma Ichthyosis Deafness Syndrome (ARKID)

Patients presented with severe palmoplantar keratoderma, ichthyosis and sensorineural deafness (Fig. 1). Exome sequencing revealed biallelic mutations in VPS33B, encoding VPS33B, which interacts with Rab11a and Rab25 proteins and is involved in trafficking of the collagen modifying enzyme lysyl hydroxylase (LH3). Patients $1 \mathrm{a}$ and $1 \mathrm{~b}$ showed the homozygous missense variant c.[390G>A;392G>A] (p.Gly131Glu), while patient 1c was compound heterozygous for p.Gly131Glu and the splice site mutation c.240-1G>C.
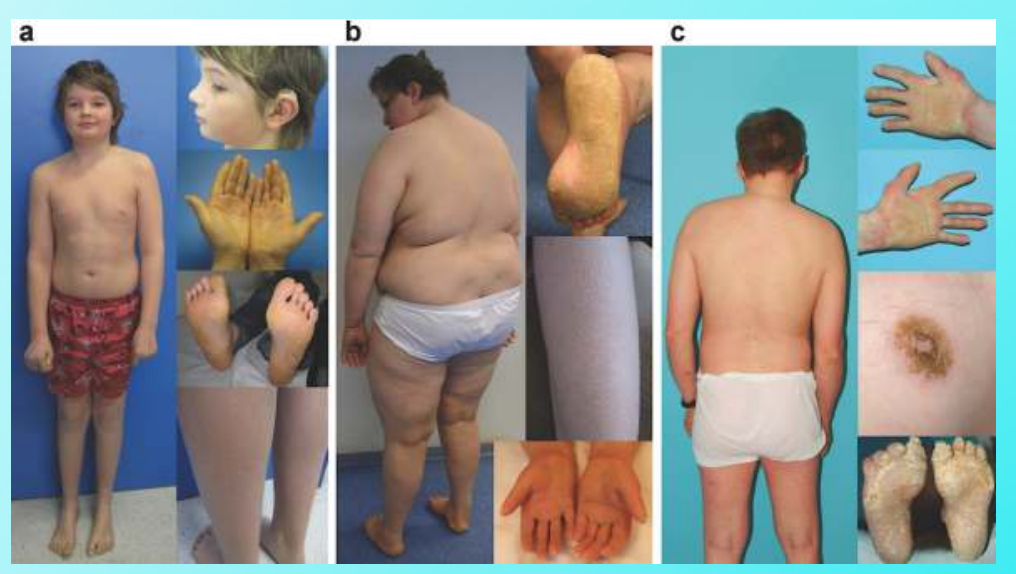

Fig. 1: (a-c)

Clinical features of ARKID patients.

VPS33B p.Gly131Glu does not affect VPS33B-VIPAR interaction Overexpressed wild-type VPS33B interacts with VIPAR (Fig. 2a). In contrast to VPS33B mutations causing arthrogryposis renal dysfunction and cholestasis (ARC) syndrome the p.Gly131Glu variant does not result in a reduced interaction with VIPAR (Fig. 2b,c), likely due to its localization (Fig. 2d).
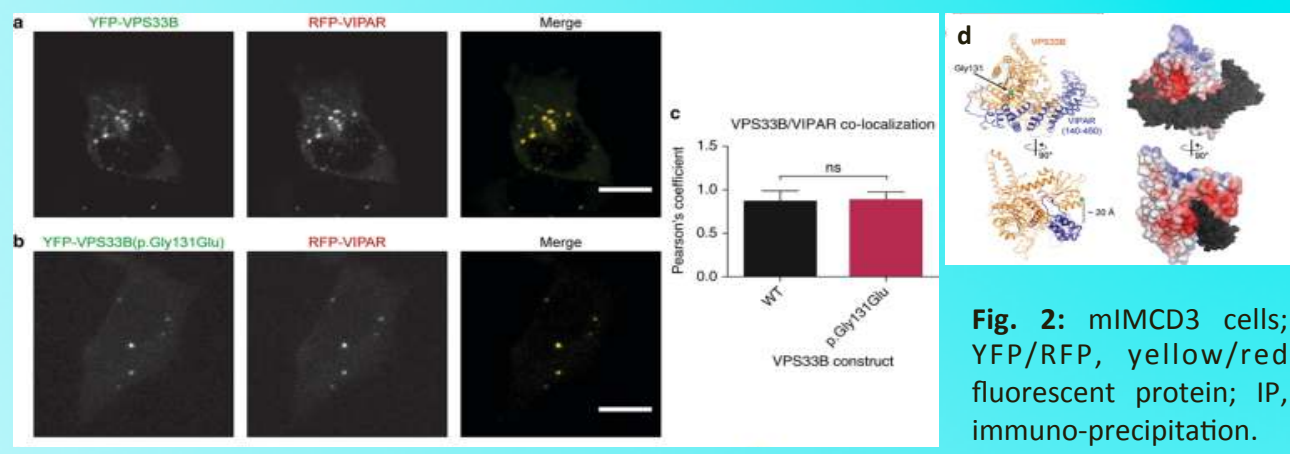

VPS33B p.Gly131Glu cannot rescue defective LH3 delivery In VPS33B-deficient mIMCD3 cells LH3 is not delivered to intracellular collagen IV carriers, however this can be rescued by cotransfection of wildtype (wt) VPS33B and VIPAR constructs into VPS33B-deficient cells. Unlike the wt construct, p.Gly131Glu mutant VPS33B cotransfected with VIPAR could not rescue LH3 trafficking (Fig. 3a). There were deficient LH3-specific collagen lysine posttranslational modifications in urine and fibroblasts of ARKID patients (Fig. 3b).

\section{a}

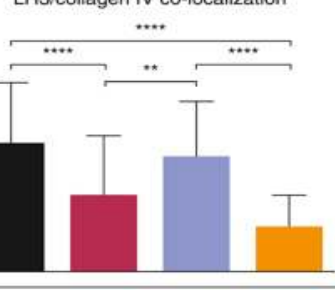

Fig. 3: (a) Quantification of LH3/collagen IV colocalization. (b,c) LC-MS-MS analysis for relative quantification of hydroxylysines (Lys-OH), galactosyl-hydroxylysines (Lys-O-Gal) and glucosylgalactosyl-hydroxylysines (Lys-O-GalGlc) from (b) urine and (c) skin fibroblasts.

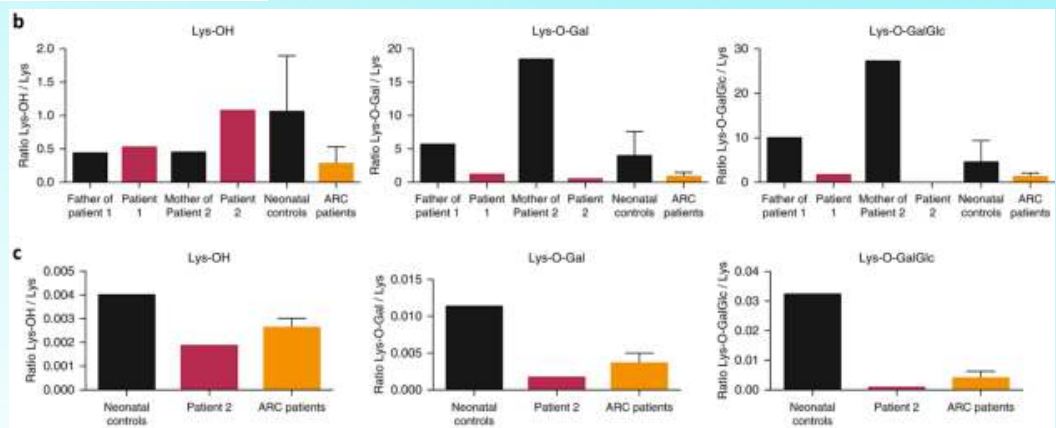

VPS33B p.Gly131Glu affects interaction of the VPS33B-VIPAR complex with Rab11 family proteins

The VPS33B-VIPAR complex interacts with both Rab25, a small GTPase necessary for LH3 delivery to intracellular collagen, and Rab11a, which is essential for epidermal lamellar body biogenesis. Compared with wt, p.Gly131Glu mutant VPS33B had markedly reduced colocalization and coimmunoprecipitation with Rab25 (Fig. 4a-c) and Rab11a (Fig. 4d-f).

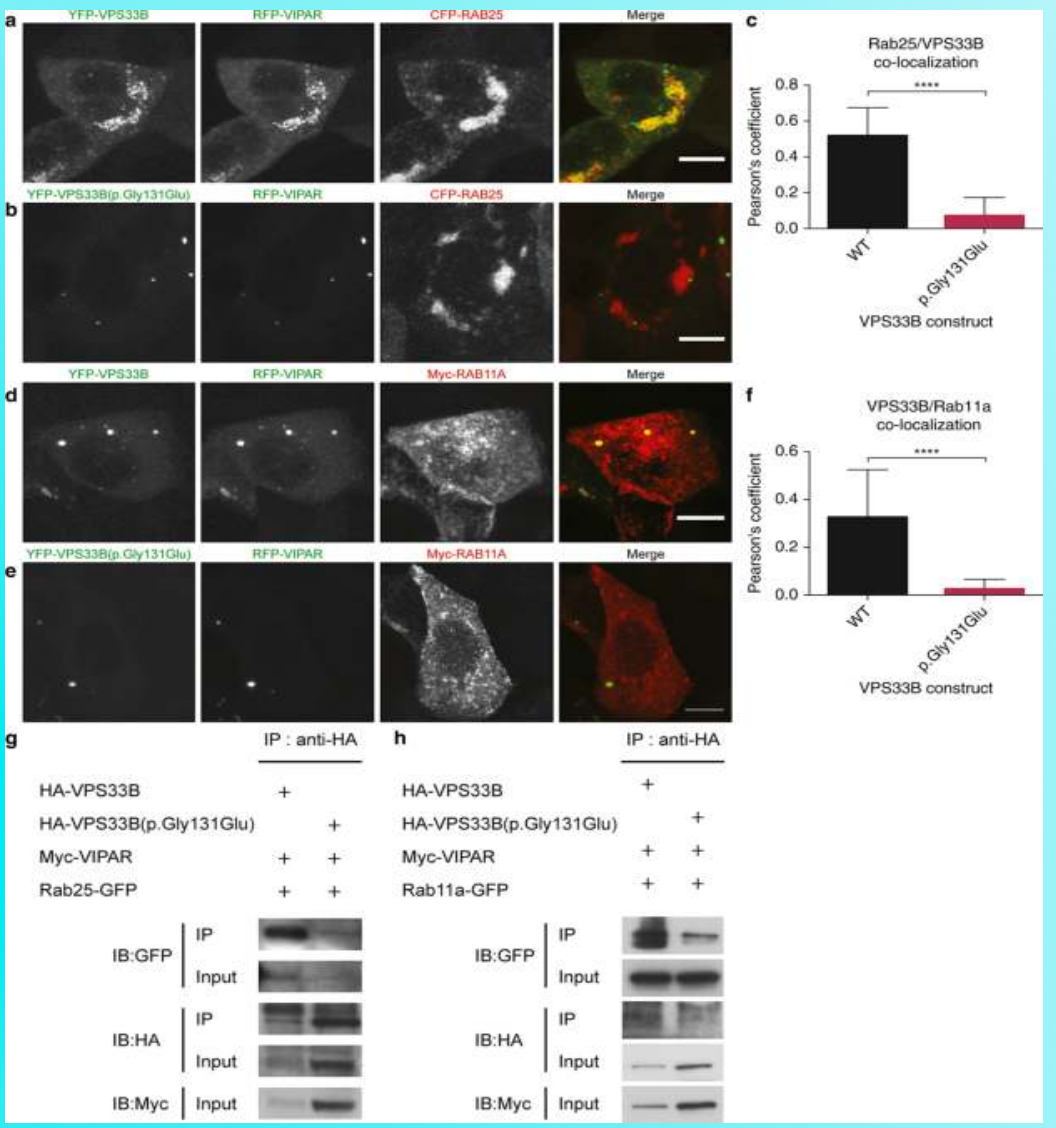

Fig. 4: (a-f) mIMCD3 cells; YFP/RFP, yellow/red fluorescent protein; IP, immunoprecipitation. (g,h) Hemagglutinin (HA) co-IP on lysates of HEK293 cells coexpressing HA-VPS33B or HA-VPS33B p.Gly131Glu with Myc-VIPAR and Rab25-GFP.

Epidermal structure of ARKID patients mirrors defects in tamoxifen-inducible VPS33B deficient Vps33 $\mathrm{b}^{\mathrm{f} / \mathrm{fl}}-\mathrm{ER}^{\mathrm{T} 2}$ mice Both patients and murine models revealed an impaired basement membrane zone and epidermal barrier structure with less prominent hemidesmosomes, a reduced number of anchoring fibrils, insufficiently fixed collagen fibers to the fibrils and aberrant secretion of lamellar bodies (LBs) (Fig. 5).

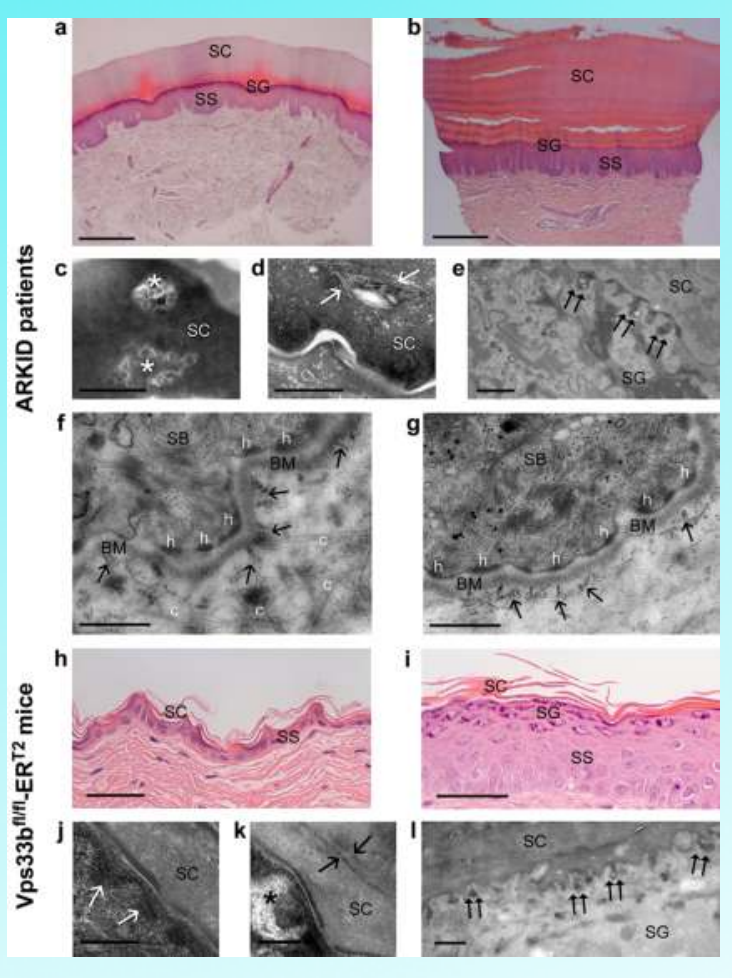

Fig. 5: (a) Control and (b) ARKID palmar skin, H\&E. (c-e) ARKID skin TEM (c) LBs (*) and (d) lipid bilayers (arrows) within corneocytes, (e) inhomogeneous LB aberrant LBs. (f, g) Dermoepidermal junction in (f) control and (g) ARKID abdominal skin, anchoring fibrils (arrows). (h) wt and (i) Vps33bf/fil-ER ${ }^{\top 2}$ dorsal epidermis, H\&E. TEM of (j) wt and (k) $V p s 33 b^{f / f l}-E R^{T 2}$ epidermis: normal (white arrows) versus entombed bilayers (black arrows), aberrant LBs $\left({ }^{*}\right)$. (I) aberrant LBs at the SG-SC interface (double arrows). secretion (double arrows) and 